\title{
Malabsorption and subtotal villous atrophy secondary to pulmonary and intestinal tuberculosis
}

W. P. FUNG, K. K. TAN, S. F. YU, AND K. M. KHO

From the Department of Clinical Medicine, University of Singapore, and the Departments of Pathology and Radiology, General Hospital, Singapore

SUM MARY A case of malabsorption and subtotal villous atrophy secondary to pulmonary and intestinal tuberculosis is reported. The patient was a 21-year-old Chinese girl who had active pulmonary tuberculosis, malabsorption, subtotal villous atrophy, atrophic gastritis with hypochlorhydria, ileal stricture, and a severe non-specific anaemia. There was also evidence to suggest protein-losing enteropathy. The association of subtotal villous atrophy and atrophic gastritis with tuberculosis is discussed. When antituberuclous therapy was instituted, improvement was marked not only clinically but also in the tests for intestinal absorption and in the jejunal mucosa.

Before the advent of antituberculous therapy, the incidence of intestinal involvement in the presence of pulmonary tuberculosis ranged from 2.8 to $90 \%$ in various $x$-ray and necropsy series (Paustian, 1964; Blumberg, 1928; Bockus, 1944; Cullen, 1940; Williams, 1939). Intestinal involvement in pulmonary tuberculosis was thus quite frequent in the pre-chemotherapy period. With the discovery of antituberculous drugs, intestinal tuberculosis became less common, and an incidence of only $1 \%$ in patients with pulmonary tuberculosis, regardless of the stage of the pulmonary disease, has been reported (Mitchell and Bristol, 1954). Over the past 25 years there has been a definite decline in the incidence of intestinal tuberculosis, as reflected by the scarcity of cases reported in the literature (Winter and Goldman, 1966). Although most authors agree that it is now rare in the western hemisphere (Winter and Goldman, 1966; Hawkins, 1963), intestinal tuberculosis, especially ileocaecal lesions, still occurs in the underdeveloped countries (Anscombe, Keddie, and Schofield, 1967; Anand, 1956; Ukil, 1942; Stock and $\mathrm{Li}, 1964$ ).

Malabsorption may be secondary to intestinal tuberculosis, dependent upon the extent and location of the inflammatory process in the small intestine (Paustian, 1964). As intestinal tuberculosis is now rare, secondary malabsorption is even rarer. This is the report of a case of malabsorption and subtotal villous atrophy secondary to pulmonary and intestinal tuberculosis. This is the first report of this unusual and rare manifestation of tuberculosis from Singapore, where pulmonary tuberculosis is still a very common disease.

\section{Case History}

A 21-year-old Chinese girl was admitted to hospital with abdominal pain, pallor, swollen ankles, diarrhoea, fever, weight loss, and amenorrhoea. Three months before admission, she started to have mild abdominal pain, which was generalized, not colicky and not related to meals. This was followed by the onset of diarrhoea (three to four stools a day) consisting of soft to watery stools, without blood or mucus. She then developed a low-grade fever, not associated with chills or rigors. She became increasingly pale and noticed abdominal distension, swollen ankles, and weight loss.

She had had no past illness and her diet was 
adequate. Physical examination showed that she was febrile (temperature, $101^{\circ} \mathrm{F}$ ) and very anaemic. She had moderate ankle oedema and the abdomen was slightly distended with a doughy feeling on palpation. Heart and lungs were clinically normal.

Investigations showed a haemoglobin of $7.6 \mathrm{~g} \%$, a total white count of $11,000 / \mathrm{cmm}$ (polymorphs $84 \%$, lymphocytes, $12 \%$ ), platelet count of $240,000 / \mathrm{cmm}$, and a reticulocyte count of $5.0 \%$. A peripheral blood film showed well haemoglobinized and normocytic red cells. The marrow showed normoblastic erythropoiesis, some hyperplasia, and increased iron stores. Punctate basophilia was present but lead poisoning was excluded. Haemolysis was not confirmed. The serum iron level was $111 \mu \mathrm{g} \%$ and biochemical tests of liver function were normal. Serum proteins, however, were low (serum albumin $1.6 \mathrm{~g} \%$, serum globulin $1.9 \mathrm{~g} \%$ ). Serum protein electrophoresis showed an albumin level of only $0.9 \mathrm{~g} \%$ and gamma globulin level of $1.3 \mathrm{~g} \%$.

As she had diarrhoea, stools were cultured for amoeba, salmonella, and shigella, with negative results. Malabsorption was confirmed by the results of the following tests: xylose absorption

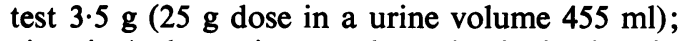
vitamin A absorption test showed a rise in vitamin A of only $96 \mathrm{IU}$ per $100 \mathrm{ml}$ serum (normal 500 and above); and a flat glucose tolerance curve. There was, however, no steatorrhoea, as stool fat excretion was only $2 \cdot 1 \mathrm{~g}$ per day. The serum potassium level was $4.6 \mathrm{~m}$-equiv/l, serum sodium $127 \mathrm{~m}$-equiv/l, serum calcium $8.5 \mathrm{mg} \%$, and serum magnesium $2.0 \mathrm{mg} \%$.

Jejunal biopsy (Fig. 1) showed a flat mucosa under the dissecting microscope, and subtotal villous atrophy with marked round cell infiltration of the lamina propria on histological sections. Gastric biopsy showed a moderate degree of atrophic gastritis with marked round cell and plasma cell infiltration of the lamina propria. There was associated hypochlorhydria (basal pH 8.0, basal acid output 0 , post-histamine pH 1.6, maximal acid output $2.4 \mathrm{~m}$-equiv/hr).

Pulmonary tuberculosis was shown by radiological examination of the chest. (A 'soft' active tuberculous lesion could be seen in the right apex.) Laryngeal swab cultures were positive for tubercle bacilli, but the Mantoux test was negative even with tuberculin dilutions of $1 / 1000$ and $1 / 100$.

Intestinal involvement was further shown by barium studies. In the barium meal and follow through, a late film showed symmetrical thickening of the ileocaecal valve, signifying oedema of this structure. The terminal ileum filled and dilated well but had irregular borders, especially on the mesenteric side. Sharp 'spikes' were also seen, suggesting oedema, lymphoid hyperplasia, and ulceration (Figure 2). A barium and air double-contrast enema showed more clearly the thickened ileocaecal valve and the irregular mesenteric border of the terminal ileum (Figure 3). A lymphangiogram showed no abnormality.

Protein-losing enteropathy was strongly suggested by the finding of clinical oedema secondary to low serum albumin levels together with the absence of proteinuria and no evidence of liver disease. The severe normocytic anaemia was associated with persistently high reticulocyte counts $(5.0 \%$ to $9.0 \%)$ in spite of no specific treatment. Haemolysis was possible but not confirmed. The anaemia was most likely secondary to the tuberculous infection since it cleared up with antituberculous therapy.

The progress of this patient is shown in Figure 5. During the initial period before therapy, she continued to have fever, diarrhoea, abdominal pain, oedema, and anaemia. Blood was given during this period and was responsible for an initial rise of haemoglobin from $7.6 \mathrm{~g} \%$ to 9.0 $\mathrm{g} \%$. After completion of most of the investigations, antituberculous therapy (streptomycin 1 $\mathrm{g}$ daily, PAS $10 \mathrm{~g}$ daily, and INAH $300 \mathrm{mg}$ daily) was started. After six days on this treatment, the fever subsided, followed by marked overall improvement. The abdominal pain, diarrhoea, oedema, and anaemia cleared up within two months of therapy and her menstrual periods returned to normal. After two and a half months of therapy, the haemoglobin had risen to $13.0 \mathrm{~g} \%$ and she had gained $15 \mathrm{lb}$ in weight. A repeat xylose absorption test showed a return to normal of $6.7 \mathrm{~g}(25 \mathrm{~g}$ dose $)$. A repeated glucose tolerance curve was normal. A jejunal biopsy was done after four weeks of antituberculous therapy, and this showed marked improvement, with leaves and fingerlike villi on dissecting microscopy (Fig. 4) and almost normal villi on histological sections. The serum albumin level returned to normal $(4.0 \mathrm{~g} \%)$, serum globulin to $2.4 \mathrm{~g} \%$, and a laryngeal-swab culture became negative for tubercle bacilli. She improved so much that she returned to work. She continues with antituberculous therapy and at present (more than a year after the onset of the illness) she has put on more weight and her health has returned to almost normal (Fig. 5).

\section{Discussion}

The history of abdominal pain preceding the diarrhoea strongly suggested some obstructive intestinal lesion and not primary malabsorption, which usually presents with diarrhoea or steatorrhoea as the initial symptom. The presence of fever also pointed to a systemic disease and when pulmonary tuberculosis was confirmed by radiology, intestinal tuberculosis had to be considered. The association of pulmonary tuberculosis with intestinal involvement has long been recorded in medical history. It was a dreaded complication in the days of Hippocrates who stated that 


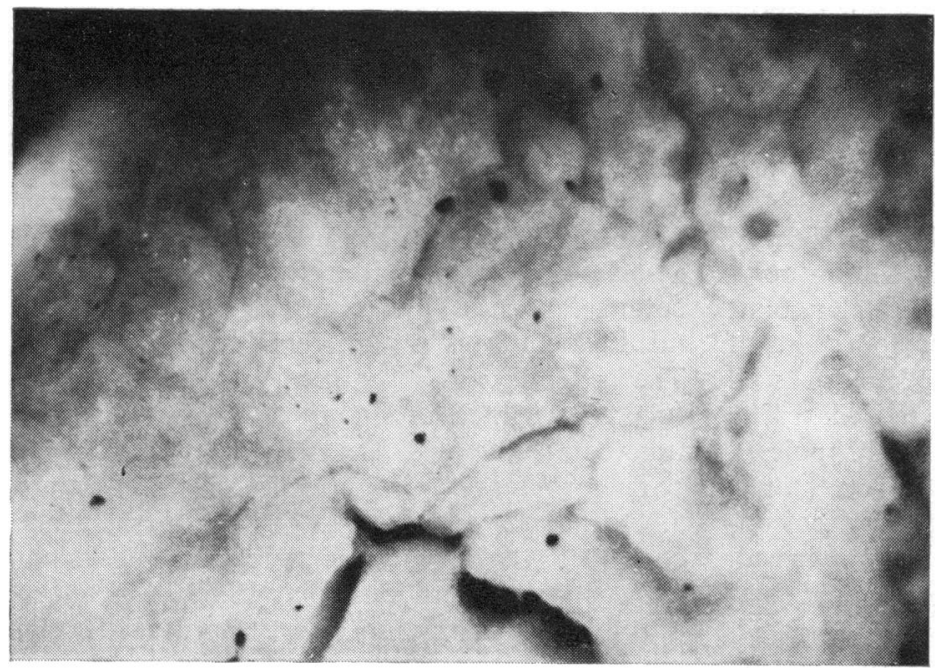

Fig. 1 Flat jejunal mucosa, as seen under the dissecting microscope, from the first jejunal biopsy, done before antituberculous therapy. Note the complete absence of any form of villi $(\times 30)$.

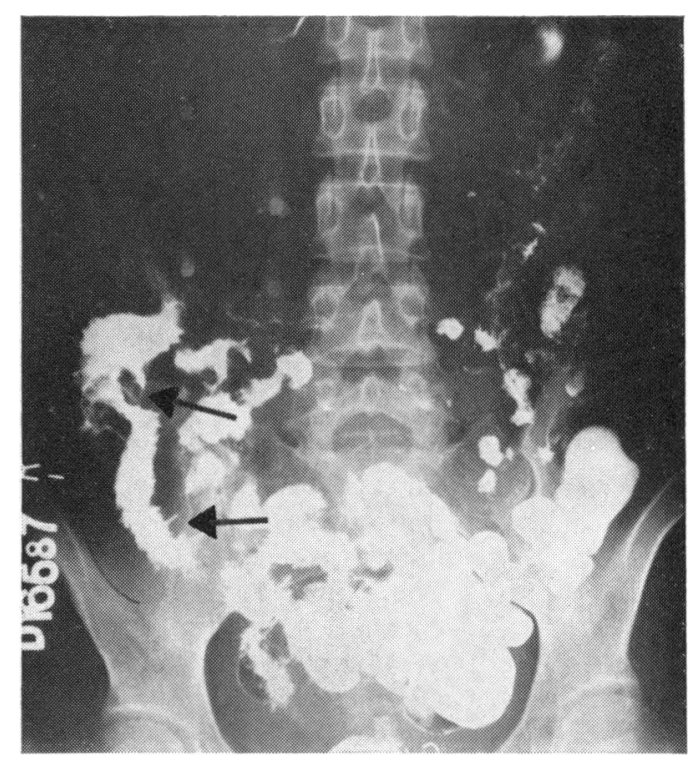

Fig. 2 A barium meal follow-through film showing the oedematous ileocaecal valve (upper arrow) and the irregular mesenteric border of the terminal ileum, which also had 'spikes' (lower arrow).

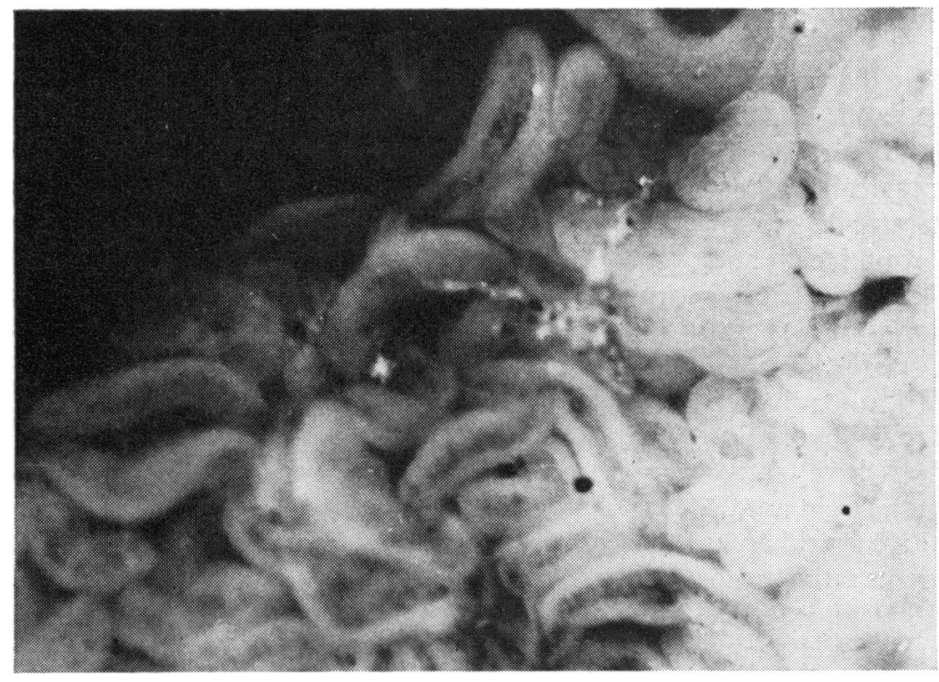

Fig. 4 Dissecting microscope appearance of the small bowel biopsy, done after four weeks of antituberculous therapy, showing marked improvement (compare with Fig. 1) with return of leaves and finger-like villi $(\times 30)$.

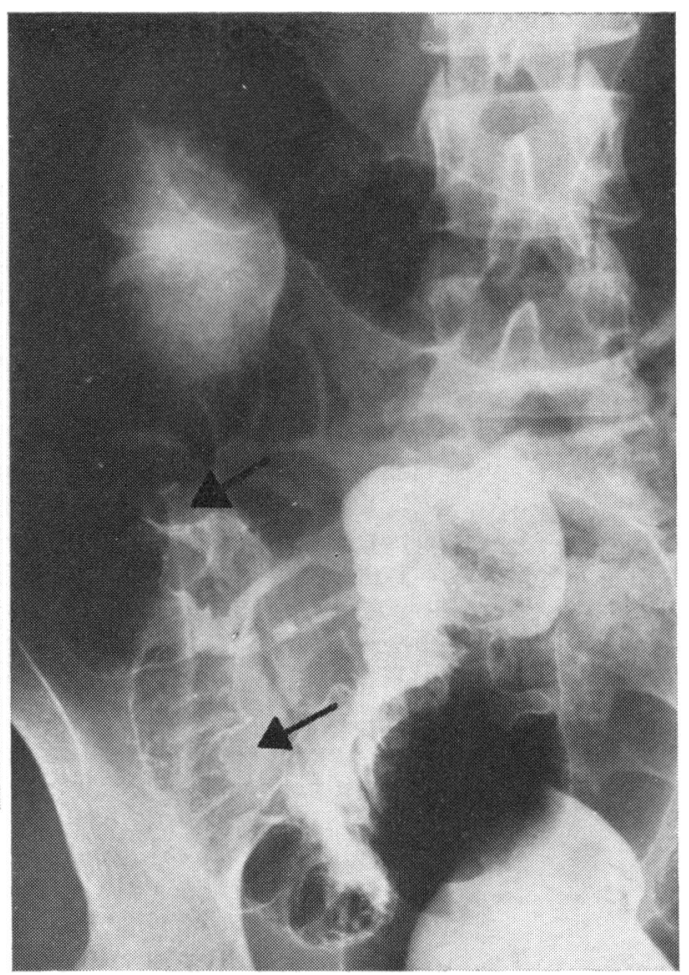

Fig. 3 A barium añ̂̀ air double-contrast enema film showing the oedematous ileocaecal valve (upper arrow) and the irregular mesenteric border of the terminal ileum (lower arrow). 
W.M.C. 2lyrs. Chinese, female.

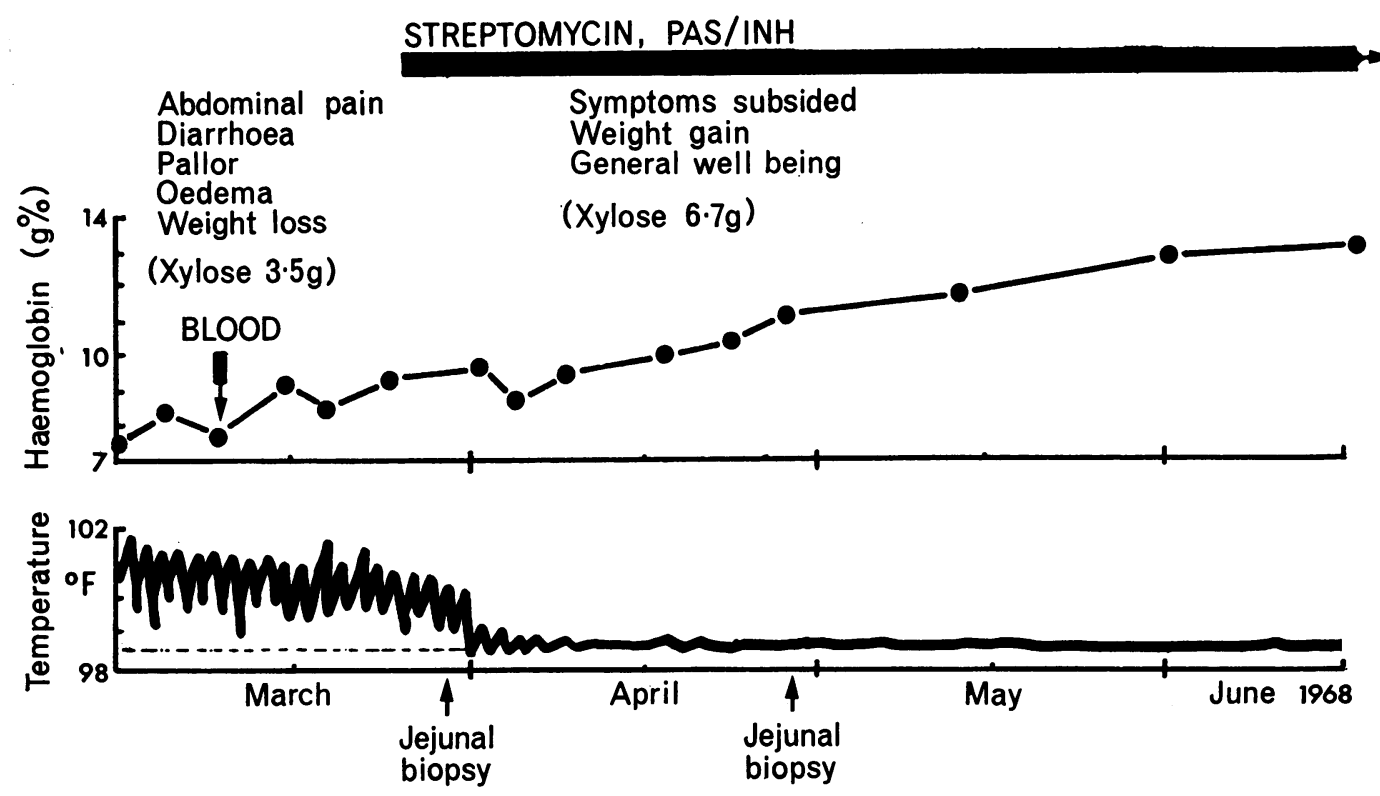

Fig. 5 Chart of clinical presentation, progress, and response to treatment. Note the marked improvement especially the subsidence of fever, rise in haemoglobin and return of xylose absorption test to normal, during antituberculous therapy.

'phthisical persons, hairs of whose head fall off, die if diarrhoea sets in ... diarrhoea attacking a person with phthisis is a mortal symptom' (Paustian, 1964).

Active pulmonary tuberculosis was confirmed in this case by positive cultures and radiology. The intestinal lesion was clearly demonstrated by the barium studies which showed the ileocaecal involvement. Further, the small intestine was also involved since there was subtotal villous atrophy with severe jejunitis, as shown by the pre-treatment jejunal biopsy. Although there was no proof of the tuberculous nature of these lesions, there was much evidence to suggest strongly this to be the case. First, the patient had proven active pulmonary tuberculosis. Secondly, the abdominal pain and chronic diarrhoea cleared up with antituberculous therapy. Thirdly, malabsorption was confirmed and there was definite improvement in absorptive function with antituberculous therapy. Finally, there was marked and prompt improvement in the jejunal mucosa as shown by the return to normal villous structure after only four weeks of antituberculous therapy.

The subtotal villous atrophy, as seen in this case, was most likely secondary to the tuberculous infection and is a further example of villous atrophy secondary to various disease processes. This finding also adds support to the contention that a flat jejunal mucosa is not absolutely specific for idiopathic steatorrhoea but can be secondary to various diseases (Hindle and Creamer, 1965). It is noteworthy that in the series of Hindle and Creamer, there was also a flat intestinal mucosa in a case of tuberculous lymphadenopathy with malabsorption.

In spite of the statement that a preoperative diagnosis of intestinal tuberculosis is unlikely (Winter and Goldman, 1966), this report shows that it can be done if adequate investigations, especially barium studies and jejunal biopsy, are carried out.

The presence of 'spiking', as seen in the barium study of this case, is not unlike that seen in Crohn's disease. In fact, many cases, diagnosed by older writers as ileocaecal tuberculosis and based on the presence of giant cells rather than tubercle bacilli, were Crohn's disease (Hawkins, 1963). However, the true nature of Crohn's disease still remains unclear.

The severe anaemia in this case was most probably secondary to the tuberculous infection since it cleared up with antituberculous therapy (haemoglobin from $7.6 \mathrm{~g} \%$ to $13.0 \mathrm{~g} \%$ ). Target cell anaemia has been reported in ileocaecal tuberculosis (McCabe, 1952).

Another interesting feature was the negative 
result of the Mantoux test. Although this is most unusual, it can occur in intestinal tuberculosis and may even be negative in the early stages of tuberculous peritonitis (Hawkins, 1963). A negative Mantoux test therefore does not exclude intestinal tuberculosis.

The possibility of protein-losing enteropathy in this patient was, unfortunately, not confirmed as isotope facilities were not available. The presence of oedema and marked hypoproteinaemia, in the absence of proteinuria and liver disease, however, strongly suggests it. Intestinal lymphangiectasia was excluded by the normal lymphangiogram and the jejunal biopsy. Atrophic gastritis and hypochlorhydria were shown in this case. In another case of pulmonary tuberculosis with gastric involvement, marked atrophic gastritis (gastric biopsy) and achlorhydria were found. It would appear that tuberculosis may be another aetiological factor in the pathogenesis of atrophic gastritis.

In conclusion, it should be emphasized that intestinal involvement and malabsorption should be looked for in every case of pulmonary tuberculosis presenting with abdominal pain and diarrhoea.

The authors are extremely grateful to Professor C. C. Booth for contributions to the discussion of this case in a clinico-pathological meeting; to Professor O. T. Khoo for permission to publish the case; to Dr S. B. Kwa for haematological investigations; to Dr E. Jacobs for the biochemical tests of intestinal absorption; and to Mr L.S. Tan for the photographs.
References

Anand, S. S. (1956). Hypertrophic ileo-caecal tuberculosis in India, with a record of $\mathbf{5 0}$ hemicolectomies. Ann. roy. Coll. Surg. Engl., 19, $205-222$.

Anscombe, A. R., Keddie, N. C., and Schofield, P. F. (1967) Caecal tuberculosis, Gut, 8, 337-343.

Blumberg, A. (1928). Pathology of intestinal tuberculosis, $J$. Lab. clin. Med., 13, 405-412.

Bockus, H. L. (1944). Tuberculosis of the intestine. In Gastroenterology, Vol. 2. Saunders, Philadelphia.

Cullen, J. H. (1940). Intest inal tuberculosis: a clinical pathological study. Quart. Bull. Sea View Hosp., 5, 143-160.

Hawkins, C. F. (1963). Diseases of the Alimentary Tract. Heinemann, London.

Hindle, W., and Creamer, B. (1965). Significance of a flat smallintestinal mucosa, Brit. med. J., 2, 455-458. Clinical picture, 458-459.

McCabe, E. S. (1952). Ileocecal tuberculosis with target cell anemia, Amer. J. dig. Dis., 19, 113-115.

Mitchell, R. S., and Bristol, L. J. (1954). Intestinal tuberculosis: an analysis of 346 cases diagnosed by routine intestina radiography on 5,529 admissions for pulmonary tuberculosis, 1924-49. Amer. J. med. Sci., 227, 241-249.

Paustian, F. F. (1964). Tuberculosis of the intestines. In Gastroenterology, edited by A. L. Bockus, Vol. 2, pp. 311-334. Saunders, Philadelphia.

Stock, F. E., and Li, F. W. P. (1964). Granulomas of the large bowel simulating malignant disease. Brit. J. Surg., 51, 898-901.

Ukil, A. C. (1942). Early diagnosis and treatment of intestinal tuberculosis. Ind. med. Gaz., 77, 613-620.

Williams, H. B. (1939). Intestinal tuberculosis: a report of 3,693 cases studied by $\mathrm{x}$-ray and at autopsy. Med. Bull. Veterans Adm. (Wash.), 15, 236-239.

Winter, J., and Goldman, M. (1966). Tuberculosis of the termina ileum. Gut, 7, 478-480. 